Repères

\title{
Le système éducatif italien
}

\section{Teresa Longo}

\section{CpenEdition}

\section{Journals}

Édition électronique

URL : http://journals.openedition.org/ries/1581

DOI : $10.4000 /$ ries. 1581

ISSN : 2261-4265

Éditeur

Centre international d'études pédagogiques

Édition imprimée

Date de publication : 1 avril 2005

Pagination : 137-144

ISSN : $1254-4590$

Référence électronique

Teresa Longo, "Le système éducatif italien », Revue internationale d'éducation de Sèvres [En ligne], 38 | avril 2005, mis en ligne le 01 avril 2008, consulté le 19 avril 2019. URL : http:// journals.openedition.org/ries/1581 ; DOI : 10.4000/ries.1581

Ce document a été généré automatiquement le 19 avril 2019

(c) Tous droits réservés 


\title{
Repères \\ Le système éducatif italien
}

\author{
Teresa Longo
}

1 Le système éducatif italien se développe à partir d'une multiplicité de repères culturels. Ceux-ci créent la richesse, mais aussi les problèmes d'un pays dont l'unité nationale s'est faite relativement tard par rapport aux autres pays européens.

2 Dans les villes du centre nord, à partir du Moyen Age, les corporations de métier avaient développé et cultivé des savoirs importants qui accompagnaient la vie quotidienne des citadins. L'urbanisme, le décor, les techniques de construction des établissements étaient riches d'un savoir et d'un goût que les habitants partageaient. La culture ainsi que la science qui s'épanouissait dans les universités attiraient l'intérêt de nombreux intellectuels européens et contribuaient au prestige de chaque ville. Les princes s'occupaient de l'instruction du peuple : en Toscane, à la fin des années 1700, le Granduca lançait, dans le cadre d'une réforme de l'agriculture, un programme d'instruction élémentaire et de formation pour tous.

3 L'Église a contrôlé, jusqu'en 1870, l'ensemble de l'Italie centrale, où seules étaient présentes dans le domaine de la culture et de l'éducation les grandes institutions catholiques. Peu nombreux étaient les hommes qui pouvaient y accéder et aucun programme d'instruction ne touchait le peuple qui vivait dans un état d'ignorance.

4 L'Italie du Sud a eu un développement culturel inégal: d'un côté des villes comme Palerme, Naples, où vivaient une aristocratie et une bourgeoisie ouvertes sur la Méditerranée et imprégnées des cultures espagnole, française et arabe et de l'autre des zones rurales où les propriétaires terriens ont empêché toute instruction des paysans pour en perpétuer la condition servile.

\section{L'histoire de l'école publique}

5 Jusqu'à la période qui a suivi la seconde guerre mondiale, la variété des langues, dialectes cultures, savoirs et modes de vie caractérisait l'Italie. Au moment de l'unification (1861), il y avait en Italie cent quinze groupes dialectaux et presque vingt groupes linguistiques. 
À la même époque, $75 \%$ de la population de l'ensemble du pays était analphabète ; au Sud, ils étaient $90 \%$.

6 La Loi du Piémont de 1859, devenue loi nationale, prévoyait l'obligation et la gratuité scolaire pour le premier cycle élémentaire (quatre ans). Jusqu'en 1911, les communes ont pris en charge la mise en place de l'obligation scolaire, tandis que le pouvoir central permettait à un nombre restreint de jeunes de fréquenter l'école secondaire.

\section{L'époque fasciste (1922-43)}

7 En 1923, une importante loi réorganisait ainsi le système scolaire : une école élémentaire de cinq ans, suivie soit par trois années d'école à caractère professionnel soit d'une école secondaire organisée en filières: le Gymnase-Lycée, la voie «noble»; le lycée scientifique; l'institut technique; le lycée d'éducation féminine. À l'exception de cette dernière (limitée à trois ans), toutes ces filières duraient huit ans. L'ordre fasciste attribuait à l'école primaire la fonction d'éducation morale, au lycée celle de formation des élites par une instruction littéraire et juridique et aux instituts techniques le rôle de formation des travailleurs manuels. La philosophie de l'idéalisme, fortement antipositiviste, donnait, dans tous les parcours scolaires, une place très réduite à l'instruction scientifique

8 Après le Concordat entre l'état fasciste et l'Église (1929), l'enseignement de la religion catholique devenait obligatoire à tous les niveaux de l'école. Cela renversa la prédominance laïque qui avait dominé depuis 1870 quand l'État avait décrété que l'enseignement religieux devait être dispensé seulement à ceux qui le demandaient, avant de le supprimer définitivement en 1877.

9 Le système scolaire de l'école fasciste était centralisé et basé sur des hiérarchies très rigides qui contrôlaient la morale et les idées des enseignants et des élèves.

10 Le climat de répression culturelle changea après la chute du fascisme, mais il faudra attendre plusieurs décennies pour que la gestion du système devienne plus démocratique.

\section{Après 1945 la démocratisation de l'enseignement}

11 L'article 34 de la Constitution républicaine (1948) affirme que « l'école est ouverte à tous. L'instruction inférieure d'une durée d'au moins huit ans est obligatoire et gratuite ; ceux qui ont les capacités et le mérite, même s'ils n'ont pas le moyen, ont le droit d'arriver aux niveaux les plus élevés de l'instruction ".

12 La définition législative et la mise en place de ces principes a été plutôt difficile, à cause d'une instabilité politique chronique ; il suffit de penser que quarante-neuf ministres de l'Instruction se sont succédé de 1946 à 1989. Jusqu'à nos jours, aucun gouvernement n'a pu réformer l'école secondaire dont la structure à présent, reste encore celle de 1923.

13 Toutefois, en 1962, le Parlement réussit à adopter la reforme du secondaire inférieur. Celle-ci organise l'obligation scolaire (jusqu'à quatorze ans), dans une structure formée par une école primaire d'une durée de cinq ans et une école media unica d'une durée de trois ans.

14 Les initiatives de réforme continuèrent pendant toute la décennie. En 1971, la Loi $n^{\circ} 820$ introduisait un «temps long » de quarante heures par semaine dans l'école élémentaire, 
ce qui représentait une révolution pour l'Italie où la tradition de la femme au foyer permettait le fonctionnement d'une école de quatre heures par jour.

En 1974, la gestion des établissements scolaires est ouverte aux associations de parents d'élèves, aux syndicats, aux représentants des autorités locales. Les mêmes décrets attribuaient aux écoles le pouvoir d'organiser sous la supervision du ministère, des activités expérimentales concernant les contenus, les méthodes et la structure.

Entre 1977 et 1979, le gouvernement change les programmes de l'école media: le latin n'est plus obligatoire, les contenus des programmes sont mis à jour et l'évaluation n'est plus faite par des notes chiffrées mais par des appréciations sur les savoirs et savoir-être de l'enfant. Au cours de cette même période, le gouvernement décide l'intégration des handicapés dans les établissements scolaires.

À partir de 1974, les régions et les communes développent un service de crèche pour les enfants de zéro à trois ans et l'école maternelle est réformée dans son organisation et ses programmes. Désormais, à la maternelle, deux enseignants s'occupent d'une classe et planifient les activités pédagogiques.

La crise économique, la diminution de la dépense publique et le terrorisme qui se diffuse dans le pays en le déstabilisant politiquement bloquent alors le processus de reforme.

\section{Les réformes}

Dans les années quatre-vingt-dix, la situation économique s'améliore. Une importante action de la justice provoque la crise des partis impliqués dans des faits de corruption. L'écroulement du bloc soviétique pousse le parti communiste à revoir son projet politique. De nouveaux partis se forment et tous s'engagent dans un processus de modernisation. Mais le sens que chacun d'entre eux donne à ce terme est différent et il implique une prise de position par rapport à la place et au rôle de l'État dans la société. Deux positions s'opposent dans les deux grandes coalitions politiques: d'un côté l'idéologie anti-étatique prônant la privatisation et la transformation de la machine étatique en entreprise ; de l'autre des positions modernistes prônant des services publics efficaces et soumis à la demande des citoyens.

À partir des années quatre-vingt-dix, les deux coalitions politiques gouvernent le pays en alternance et apportent des changements importants. En 1993, un gouvernement de "techniciens" de centre-gauche arrive à faire approuver une reforme radicale de l'administration publique : la loi Bassanini, (Loi $n^{\circ}$ 29/1993). Elle affirme les principes de la déréglementation et de la simplification procédurale de l'administration publique. La loi transfère des compétences centrales aux autorités locales et aux unités de service et réorganise les hiérarchies et l'organisation du travail de tout le secteur public.

21 Les employés publics, y compris les enseignants, perdent leur statut de fonctionnaires En 1997, les établissements scolaires obtiennent une importante autonomie de gestion pilotée par le directeur désormais devenu le dirigente.

Depuis 2001, le gouvernement de la coalition de droite, dans une optique d'«harmonisation» du secteur public et privé, a accru l'autonomie de l'école en attribuant plus de pouvoir au dirigente de l'établissement pour la gestion financière, le recrutement du personnel et l'évaluation.

23 Le ministère de l'Instruction publique a alors changé son nom et est devenu ministère de l'Instruction, perdant l'appellation « publique ». 


\section{Structure et organisation du système éducatif}

L'école maternelle qui accueille les enfants de trois à six ans est gratuite ; fréquentée par $90 \%$ des enfants, elle n'est pas obligatoire mais elle est considérée comme une étape du parcours éducatif. L'école maternelle dépend de l'administration de l'État pour ce qui concerne les enseignants; les communes s'occupent des édifices, de l'entretien et du personnel d'assistance.

L'école est organisée en groupes d'âge d'un minimum de quatorze et d'un maximum de vingt-huit enfants. Deux enseignants sont chargés du groupe dans les écoles à temps complet (huit heures par jour).

6 L'obligation scolaire est actuellement de neuf ans à partir de l'âge de six ans et elle s'accomplit dans une école élémentaire pendant cinq ans, une école secondaire inférieure pendant trois ans, la «Media Unica» et pendant la première année de l'école secondaire. Jusqu'à la Media, le programme d'études est le même pour tous.

27 L'école élémentaire, à partir de 1990, s'organisait en modules de deux classes dont l'enseignement était confié à une équipe de trois enseignants remplaçant l'enseignant unique traditionnel. L'équipe planifiait les activités et elle répartissait les taches didactiques. Ce système permettait une certaine spécialisation de l'enseignant et en même temps une continuité de sa présence dans la classe. De plus, les enseignants mettaient en synergie leurs capacités dans l'équipe responsable. En 2004, malgré les protestations des enseignants et des familles, le ministère a changé d'organisation: un enseignant «tutor» deviendra le responsable de la classe où il enseignera plusieurs disciplines pendant dix-huit à vingt heures et les autres heures reviendront à des enseignants qui circuleront entre plusieurs classes. Le travail d'équipe lié à la classe est ainsi destiné à disparaitre.

28 L'école peut durer de vingt-sept à quarante heures par semaine, mais l'école « longue » fait, aujourd'hui, l'objet d'un débat public.

La scuola media d'une durée de trois ans doit consolider les connaissances élémentaires et orienter vers des études secondaires spécialisées. L'horaire d'enseignement de trente heures hebdomadaires est partagé entre des disciplines enseignées par des enseignants spécialistes. À la fin de la Media, un examen évalue par une note les apprentissages. En 1977, un système d'évaluation basé sur l'observation et le contrôle des processus d'apprentissage était mis en place mais dix ans après il a été contesté et changé par un système qui évalue les résultats qui s'étalent sur une échelle qui va de $\mathrm{A}$ à $\mathrm{E}$.

L'école secondaire supérieure est divisée en de nombreuses filières : le lycée, les instituts techniques et les instituts professionnels de la durée chacun de cinq ans. Les études s'achèvent par un examen « maturità » qui confère un titre légal. Au lycée, l'horaire est de vingt-huit à trente heures hebdomadaires, tandis que dans les filières professionnelles le nombre d'heures est plus important. Depuis 1969, tous les jeunes qui ont obtenu une maturità peuvent s'inscrire à l'Université.

31 La structure de l'école secondaire supérieure italienne est encore celle de 1923. À chaque législature, des projets de réforme ont été présentés, mais l'accord des forces politiques et des différents groupes de pression a été impossible. Toutefois l'école secondaire a continué à fonctionner grâce aux expérimentations pédagogiques. Deux tiers des établissements ont été impliqués dans des projets d'innovation didactique et, en 1991, 
$70 \%$ des lycées et trois-quarts des instituts techniques faisaient des expérimentations de transformation de la structure de l'école et adaptaient les diplômes aux nouvelles demandes du marché du travail.

Les régions et les provinces sont en charge des établissements scolaires et d'une partie du personnel de l'école secondaire.

\section{Scolarisation et échec scolaire}

Tous les enfants italiens fréquentent l'école obligatoire mais, au Sud, un nombre important de jeunes quitte la scuola media avant la fin des études. Dans les années quatrevingt-dix, une enquête a montré que, à ce niveau-là, les taux d'abandons au Sud étaient de $1,24 \%$ et de $2 \%$ dans les îles tandis que la moyenne des abandons au Nord était de $0,13 \%$ (Dei, 2000).

Le problème le plus important de scolarisation se présente au niveau de l'école secondaire. En 2000, la moyenne européenne des jeunes de 25/34 ans sans diplôme secondaire étant de $29 \%$ (Eurydice, 2002) ; ce pourcentage en Italie atteignait $41 \%$; si l'on considère les Italiens âgés de 55 à 64 ans, ce pourcentage arrive à $76 \%$.

La scolarisation et la réussite dans l'école secondaire sont fortement marquées par l'origine sociale. Si l'on considère la dernière classe du secondaire, sur cent jeunes dont le père appartient à la catégorie des cadres, 73,3 sont inscrits au lycée et les autres dans les Instituts techniques; cette proportion se renverse pour les familles ouvrières parmi lesquelles seulement 22,4\% fréquentent les lycées et 77,6 les instituts techniques (ISFOL 1999).

Dans le parcours scolaire, l'année la plus difficile est la première de l'école secondaire supérieure. En 1998, à la fin cette classe, environ 6,7\% des inscrits abandonnaient les études et $18,5 \%$ redoublaient l'année ( $24 \%$ dans les instituts professionnels). Par contre, ceux qui arrivent en dernière année de secondaire ont une bonne chance de réussir l'examen final, le taux de passage à la maturità se situant autour de 97 \% (Dei, 2000).

La plupart des diplômés du secondaire continuent les études à l'université, mais $25 \%$ des étudiants de la première année abandonnent les études et seulement quatre étudiants sur dix réussissent à obtenir un diplôme au bout de six années (Istat-Murst 2003).

\section{Débats actuels}

\section{Le faible besoin de main d'œuvre qualifiée}

L'économie italienne demande peu de main d'œuvre qualifiée. À la fin des années quatrevingts, l'Italie occupait la cinquième place parmi les pays industrialisés, mais l'importance des industries «science based » diminue et la demande de force de travail qualifiée est très faible. Sur cent travailleurs, seulement 5,7 ont un titre universitaire et ces travailleurs sont surtout dans le secteur des services. La petite industrie manufacturière qui produit pour l'exportation et contribue à la croissance du pays n'a pas besoin de main d'œuvre qualifiée. Le débat actuel concerne la perpétuation de ce modèle ou bien un changement dans le cadre de l'intégration européenne, par une intervention des pouvoirs publics. 


\section{La réforme permanente et inachevée du système scolaire}

39 L'instabilité politique du pays pèse sur les possibilités de faire face aux problèmes de l'école. L'histoire récente de la réforme de la structure et des programmes le montre assez bien. Comme on l'a dit, aucun gouvernement n'a été capable, depuis soixante ans, de mener à son terme la réforme de l'école secondaire. La comparaison avec les autres pays d'Europe a mis en évidence que l'Italie avait l'une des durées les plus courtes de scolarité obligatoire. En 1999, une réforme élevait donc la durée de l'obligation scolaire à neuf ans et organisait l'école obligatoire selon une structure en cycles, éliminant du même coup la Media. Mais en 2001, les initiatives des écoles pour la mise en place de la réforme étaient bloquées par le ministre de la nouvelle coalition politique au pouvoir. Silvio Berlusconi, Président du conseil, nomme alors ministre de l'Éducation Letizia Moratti ${ }^{1}$ qui, en mars 2003, fait approuver par le Parlement une loi précisant que le droit (et non plus l'obligation) à l'instruction et à la formation est assuré pour une durée de douze années (cinq ans à l'école primaire, trois ans au secondaire inférieur et quatre au secondaire supérieur). Le système de formation professionnelle est intégré au système scolaire et la Scuola media est de nouveau présente.

La loi propose à une commission d'élaborer un curriculum flexible, basé sur des critères définis au niveau national. Les décisions de la commission concernant les contenus ont soulevé des critiques dans la société italienne, notamment sur l'élimination de l'étude de la théorie de l'évolution dans l'école obligatoire.

\section{L'école à plein temps en question}

41 La décision de diminuer le volume horaire annuel dans l'école primaire ouvre un débat public aux nombreux enjeux sociaux, culturels et idéologiques concernant le rapport entre les familles et le service public de l'éducation.

Traditionnellement les élèves italiens ne fréquentaient l'école que pendant la matinée ; la famille devait se charger du reste du temps dans la journée. L'émancipation des femmes, leur présence plus importante dans le monde du travail a conduit à prolonger le service scolaire. Les mouvements politiques pour la démocratisation de l'école se sont également prononcés pour une école à plein temps. En 1967, selon Don Milani dans la Scuola di Barbiana, « la vieille école était faite pour les classes aisées d'abord du fait de ses horaires et de son calendrier. La nouvelle école ne les a pas changés. Elle reste une culture pour les riches... Mais il y a une espérance : l'école à temps plein » (p. 31). Les écoles à plein temps ont été mises en place dans les années soixante-dix mais avec des ressources assez limitées qui ne permettaient pas partout les innovations pédagogiques.

43 Entretemps, dans le pays, s'est développée une offre particulière d'activités sportives, culturelles et de formation qui entre en concurrence avec l'école. Dans une partie des familles aisées où souvent la mère ne travaille pas, on revendique la liberté de choix dans le temps de l'après-école. Le débat se transforme souvent en une confrontation entre deux idéologies: d'une part la défense de la scolarisation publique, d'autre part, la défense de l'éducation familiale par les mouvements intégristes catholiques. Les contraintes budgétaires donnent finalement raison à ces derniers. 
INDEX

Mots-clés : système éducatif

Index géographique : Italie

AUTEUR

TERESA LONGO

Professeur à l'université Jules Verne, Amiens. 